\title{
Real world scenarios in rare variant association analysis: the impact of imbalance and sample size on the power in silico
}

Xinyuan Zhang ${ }^{1}$, Anna O. Basile ${ }^{2}$, Sarah A. Pendergrass ${ }^{3}$ and Marylyn D. Ritchie ${ }^{1,4^{*}}$

\begin{abstract}
Background: The development of sequencing techniques and statistical methods provides great opportunities for identifying the impact of rare genetic variation on complex traits. However, there is a lack of knowledge on the impact of sample size, case numbers, the balance of cases vs controls for both burden and dispersion based rare variant association methods. For example, Phenome-Wide Association Studies may have a wide range of case and control sample sizes across hundreds of diagnoses and traits, and with the application of statistical methods to rare variants, it is important to understand the strengths and limitations of the analyses.
\end{abstract}

Results: We conducted a large-scale simulation of randomly selected low-frequency protein-coding regions using twelve different balanced samples with an equal number of cases and controls as well as twenty-one unbalanced sample scenarios. We further explored statistical performance of different minor allele frequency thresholds and a range of genetic effect sizes. Our simulation results demonstrate that using an unbalanced study design has an overall higher type I error rate for both burden and dispersion tests compared with a balanced study design. Regression has an overall higher type I error with balanced cases and controls, while SKAT has higher type I error for unbalanced case-control scenarios. We also found that both type I error and power were driven by the number of cases in addition to the case to control ratio under large control group scenarios. Based on our power simulations, we observed that a SKAT analysis with case numbers larger than 200 for unbalanced case-control models yielded over $90 \%$ power with relatively well controlled type I error. To achieve similar power in regression, over 500 cases are needed. Moreover, SKAT showed higher power to detect associations in unbalanced casecontrol scenarios than regression.

Conclusions: Our results provide important insights into rare variant association study designs by providing a landscape of type I error and statistical power for a wide range of sample sizes. These results can serve as a benchmark for making decisions about study design for rare variant analyses.

Keywords: Rare variant association analysis, Sample size imbalance, Power analysis, Simulation study

\footnotetext{
* Correspondence: marylyn@pennmedicine.upenn.edu

'Genomics and Computational Biology Graduate Group, Perelman School of

Medicine, University of Pennsylvania, Philadelphia, PA, USA

${ }^{4}$ Department of Genetics, University of Pennsylvania, Perelman School of

Medicine, Philadelphia, PA, USA

Full list of author information is available at the end of the article
}

(c) The Author(s). 2019 Open Access This article is distributed under the terms of the Creative Commons Attribution 4.0 International License (http://creativecommons.org/licenses/by/4.0/), which permits unrestricted use, distribution, and reproduction in any medium, provided you give appropriate credit to the original author(s) and the source, provide a link to the Creative Commons license, and indicate if changes were made. The Creative Commons Public Domain Dedication waiver (http://creativecommons.org/publicdomain/zero/1.0/) applies to the data made available in this article, unless otherwise stated. 


\section{Background}

During the last decade, Genome-Wide Association Studies (GWAS) have greatly advanced our understanding of the impact of common variants on complex traits. The associations of alleles with frequency more than 1-5\% have provided important insights into research and clinical practice $[1,2]$. Despite GWAS revealing novel disease associations, limited genetic heritability has been explained by GWAS results [3]. Rare alleles, with moderately large genetic effect sizes, may explain more of the phenotypic variance of complex disease [4]. Low frequency or rare variants may have an essential contribution to unexplained missing heritability $[5,6]$. The development of sequencing technologies has increased access to rare variation data for large sample sizes. However, it is crucial to better understand the statistical power and analytic limitations of rare variant association approaches.

Due to the low frequency of rare variants, single locus association tests in traditional GWAS are underpowered for rare variant association analysis [7] unless the casual variants have very large effect sizes [8]. To boost power, region-based collapsing or binning approaches have become a standard for analyzing rare variants [7]. These methods evaluate the association of the joint effect of multiple rare variants in a biologically relevant region with the outcome [8].

Numerous association methods have been developed [7, 9-18], and this manuscript focuses on evaluating two of the most commonly used approaches for gene-based testing, burden and dispersion, using a simulation approach. Burden tests summarize the cumulative effect of multiple rare variants into a single genetic score and test the association between this score and phenotypic groups using regression [8]. The major assumption of burden tests is that all rare variants in a group have the same direction and magnitude of effect on the trait [8], and violation of this assumption leads to a loss of power [14]. Dispersion tests, on the other hand, evaluate the distribution of genetic effects between cases and controls by applying a score-based variance-component test [8]. The sequence kernel association test (SKAT) is a widely used dispersion method. It applies a multiple regression model to directly regress the phenotype on genetic variants in a region, followed by a kernel association test on the regression coefficients [9]. SKAT is robust to the magnitude and direction of genetic effects as well as to the presence of neutral variants, or a small portion of disease variants $[8,9]$.

Statistical power for both burden and dispersion tests has been assessed in many simulation settings $[7,9,15$, 19, 20], however, these simulations have focused on an equal (or balanced) number of cases and controls. In real data scenarios, researchers often have unequal (or unbalanced) number of cases and controls. With the application of association methods on unbalanced samples, it is beneficial to acquire the expected type I error and power to guide the study design for rare variant association tests. For example, for diseases that have a low prevalence in the population, what number of cases and how many controls are necessary to detect the impact of rare variation on the disease? In Phenome-Wide Association Studies (PheWAS) [21] there are potentially a wide range of case and control numbers and overall sample sizes across hundreds of diagnoses and traits [22-24]. A challenge for PheWAS studies using rare variants is to understand the impact of varying sample sizes, varying case numbers, and genetic effect sizes [24].

In this study, we performed extensive simulation analyses to assess the influence of sample size on the type I error and power distribution for regression (a burden test) and SKAT (a dispersion test). We designed twelve balanced sample size datasets and twenty-one unbalanced sample size scenarios. Since a large sample size has been widely known as a necessity for detecting significant rare variant associations [7, 8, 25], in this paper, we mainly simulate unbalanced scenarios using a large total sample size. BioBin [26-30] was used for rare variant binning and association testing. Results on the statistical performance of both logistic regression and SKAT can serve as a benchmark for making decisions about future rare variant association studies.

\section{Results}

We evaluated burden-based tests using logistic regression and dispersion-based tests using SKAT. All associations are evaluated for a binary outcome on a simulated gene with an average of 143 rare variant loci. We varied the number of cases, controls, and also the balance between cases and controls. All reported results here have a MAF upper bound (UB) set at 0.01. The supplementary material (Additional file 1: Figures S1 and S2) shows results with a MAF upper bound (UB) of 0.05 .

\section{Type I error results}

Figure 1 displays the overall type I error simulation results for both balanced and unbalanced sample sizes. As shown in Fig. 1a, with balanced number of cases and controls, the type I error for both regression and SKAT is well controlled under 0.05 with a few exceptions (the type I error for these was still below 0.1). Interestingly, regression had an overall higher type I error rate compared with SKAT for balanced samples. In addition, SKAT had an overall slightly increased type I error as the overall sample size increased. For regression, however, with increasing overall sample size, we did not observe an overall increasing trend in the Type I error rate. 


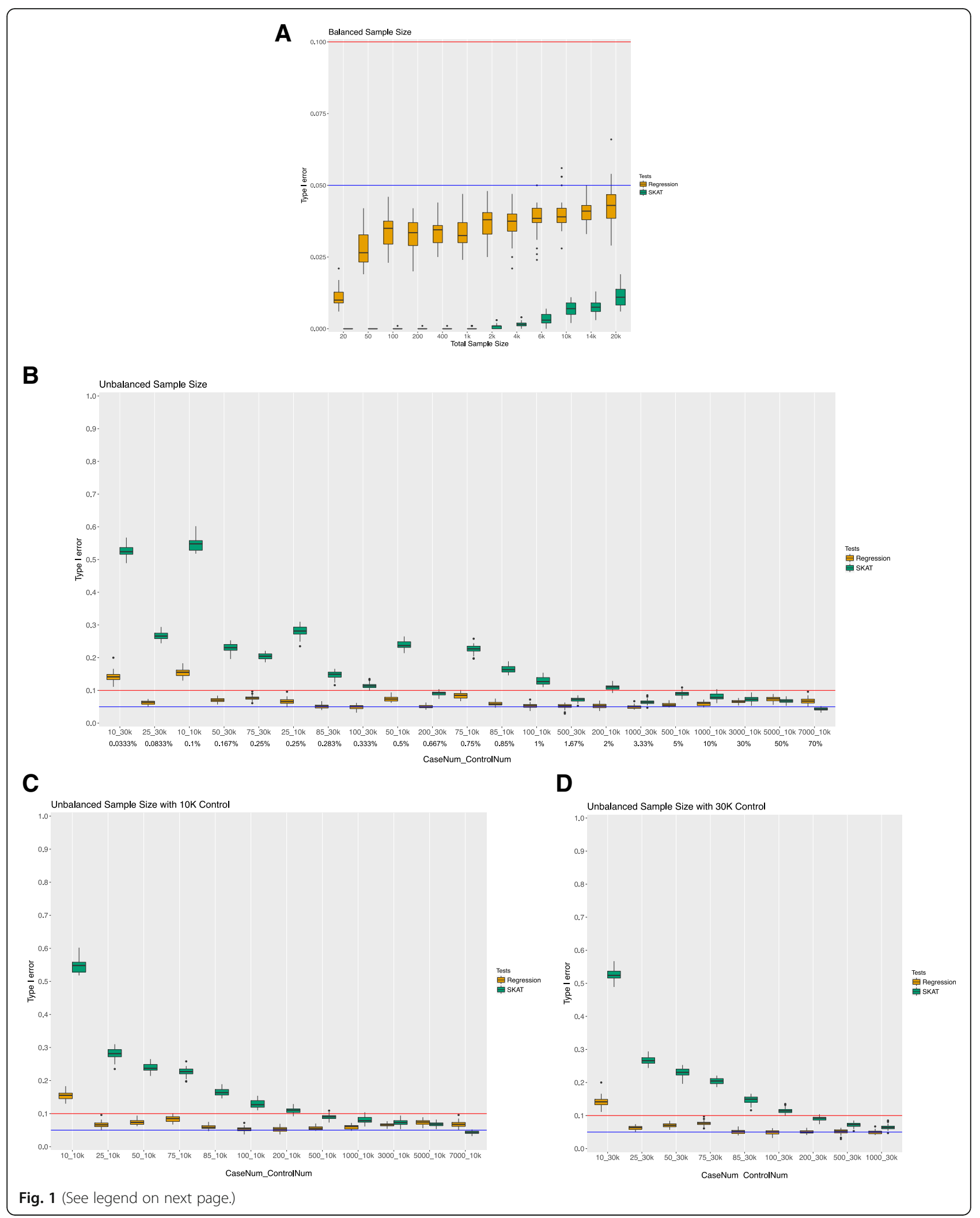


(See figure on previous page.)

Fig. 1 Type I error simulation results with MAF UB of 0.01. For visualization and comparison purposes, blue and red horizontal lines indicate type I error at 0.05 and 0.1 respectively. Fig. (a) shows the results for type I error for an equal number of cases and controls for differing sample sizes. Note that the $y$-axis only goes to a type I error rate of 0.1. Fig. (b) shows the type I error rate for different unbalanced cases and controls as arranged by case to control ratio. The axis is labeled by the number of cases then the number of controls for each simulation. The percentage of cases to controls is also listed below the number of cases and controls. Figs. (c and $\mathbf{d}$ ) show the results as ordered by the number of cases.

Figure 1c has 10,000 control and Fig. 1d has 30,000 control

Similar results have also been observed with MAF UB of 0.05 (Additional file 1: Figure S1A).

For unbalanced sample sizes, we investigated whether the type I error rate was driven by the ratio of the cases to controls or by the number of cases when having a large control sample. We ordered the sample sizes by case to control ratio in Fig. 1b, and by case number within the same control sample size in Fig. 1c and Fig. $1 d$. The type I error distribution for differing numbers of cases regardless of the number of controls had similar trends (Fig. 1c and Fig. 1d). Thus, our results suggest that number of cases tends to drive the type I error rate in addition to the case to control ratio under large control group scenarios.

An overall higher type I error rate in unbalanced case-control ratios (Fig. 1b) was observed compared to balanced case-control ratios (Fig. 1a) for both tests, most of which are higher than 0.05 . Contrary to what was seen in balanced samples, type I error rates for SKAT were overall higher than regression. An exception to this for SKAT is seen when the case number increase significantly such as 5000 and 7000 cases with 10,000 controls. Overall, for SKAT there is decreasing type I error trend as case number increases (Fig. 1c and Fig. 1d). Regression, on the other hand, has a relatively consistent type I error in the unbalanced case control ratio tests.

\section{Power results}

\section{Odds ratio 2.5}

For balanced numbers of cases and controls and an odds ratio 2.5 for rare disease loci, the power distribution is shown in Fig. 2a. The results indicate that regression has relatively higher power than SKAT for a sample size less than 1000, while SKAT has higher power given larger sample sizes $(\geq 4000)$. For a total sample size less than 2000 , both methods have less than $50 \%$ power to detect true positive effects. In order to achieve $90 \%$ power, a total balanced sample size of 4000 is needed for SKAT and nearly 14,000 is needed for regression, based on our power simulation settings.

Importantly, SKAT has an overall higher power for unbalanced cases and controls than regression (Fig. 2b). Similar to the type I error distribution, power was also driven by the number of cases instead of the ratio of cases to controls under large control group scenarios (Fig. 2b-d). Notably, overall power was improved whether tested via SKAT or regression approach with an unbalanced case control ratio compared to the balanced case control ratio simulations.

The power analyses for unbalanced samples suggest an overall increasing trend as the number of cases increases. Based on the MAF UB of 0.01 results (Fig. 2c and d), SKAT power for an unbalanced number of cases with case numbers larger than 200 does yield a mean power over 90\%. For regression with an unbalanced sample size, more than 1000 cases would yield a mean power of $90 \%$ under a 10,000 controls sample size, while case numbers more than 500 would yield the same power under a 30,000 subject control sample size. The same trend has been observed for a MAF UB of 0.05 (Additional file 1: Figure S2c and d).

\section{Mixture of genetic variation contributing to risk and protection for outcome}

The above power simulations were performed on 10 disease loci where rare variants had an odds ratio 2.5 contributing to risk. In order to better assess the performance of statistical methods, we designed three sets of models containing variants contributing to both protection and risk with varied effect sizes for 10 disease loci (see Methods for more details). We compare four scenarios here: an upper bound on simulated rare variants with a MAF of 0.01 and 0.05 ; a balanced sample size with 2000 cases and 2000 controls, and an unbalanced sample size with 200 cases and 10,000 controls. We chose these sample sizes from the results of our previous simulations as we observed both regression and SKAT to have adequate power and controlled type I error with these case control numbers.

As shown in Fig. 3, the power increases as the impact of rare variation on outcome increases. SKAT outperforms regression in all scenarios, which is expected since the power for burden tests decrease when both protective and risk effects are present. Comparing a MAF UB of 0.05 (left two plots) and a MAF UB of 0.01(right two plots) indicates that SKAT has higher power for MAF UB of 0.05 whereas regression doesn't have distinguishable power differences. When comparing the top two plots of Fig. 3 with the bottom two plots, we observe higher power for regression in unbalanced samples with 200 cases and $10 \mathrm{k}$ controls compared to 2000 cases and 


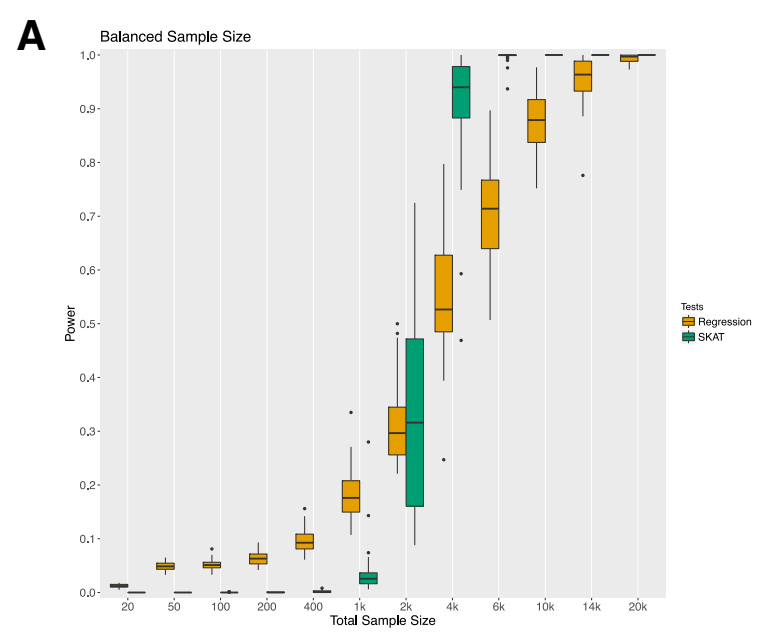

\section{B}

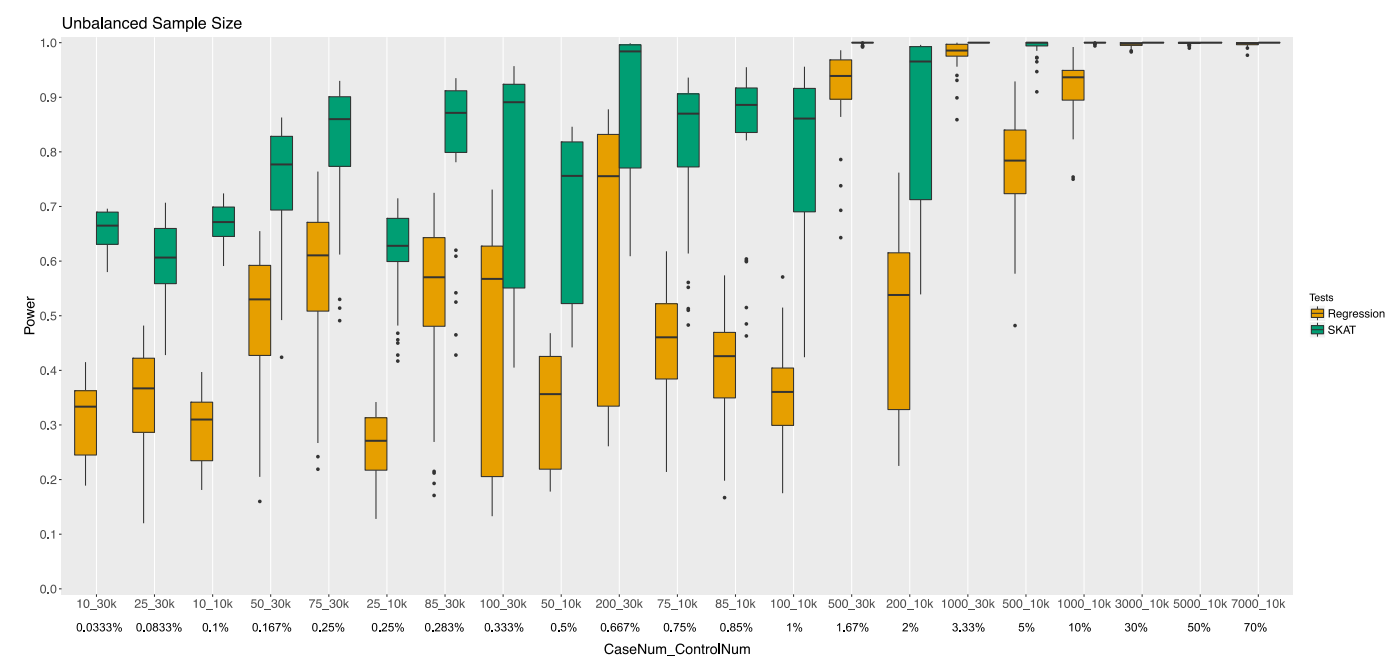

C

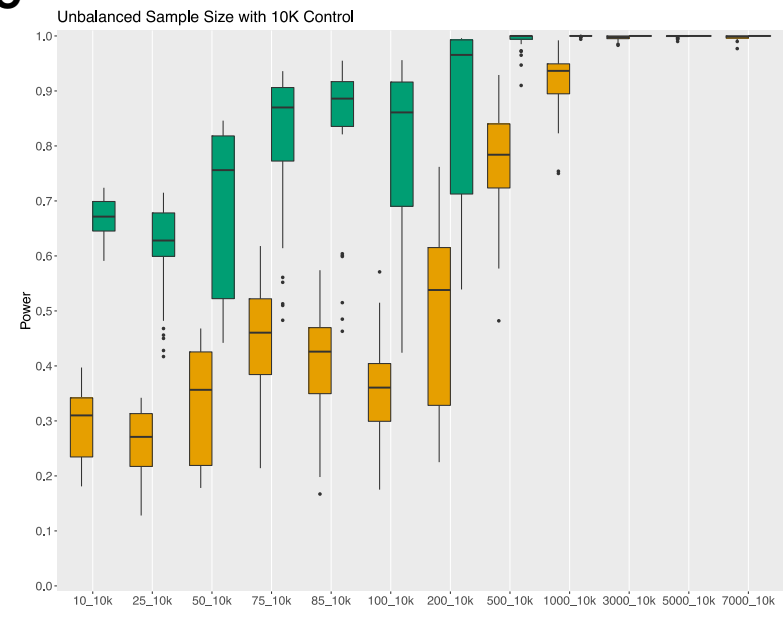

D

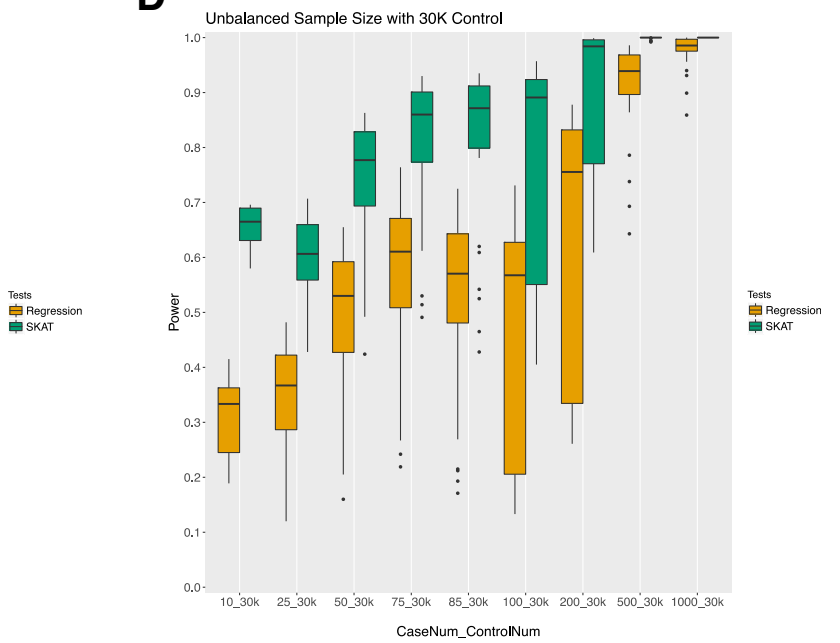

Fig. 2 Power simulation results with cutoff for evaluated variation of MAF 0.01. Fig. (a) shows the results when cases and controls are equal in number. Fig. (b) shows the impact of unbalanced cases and controls on power ranked by the case/control ratio. The percent case to control ratio is listed below the $\mathrm{x}$-axis. Figs. (c and $\mathbf{d}$ ) show the results for power with unbalanced cases and controls ordered by case number with 10,000 controls (c) and 30,000 controls (d) 

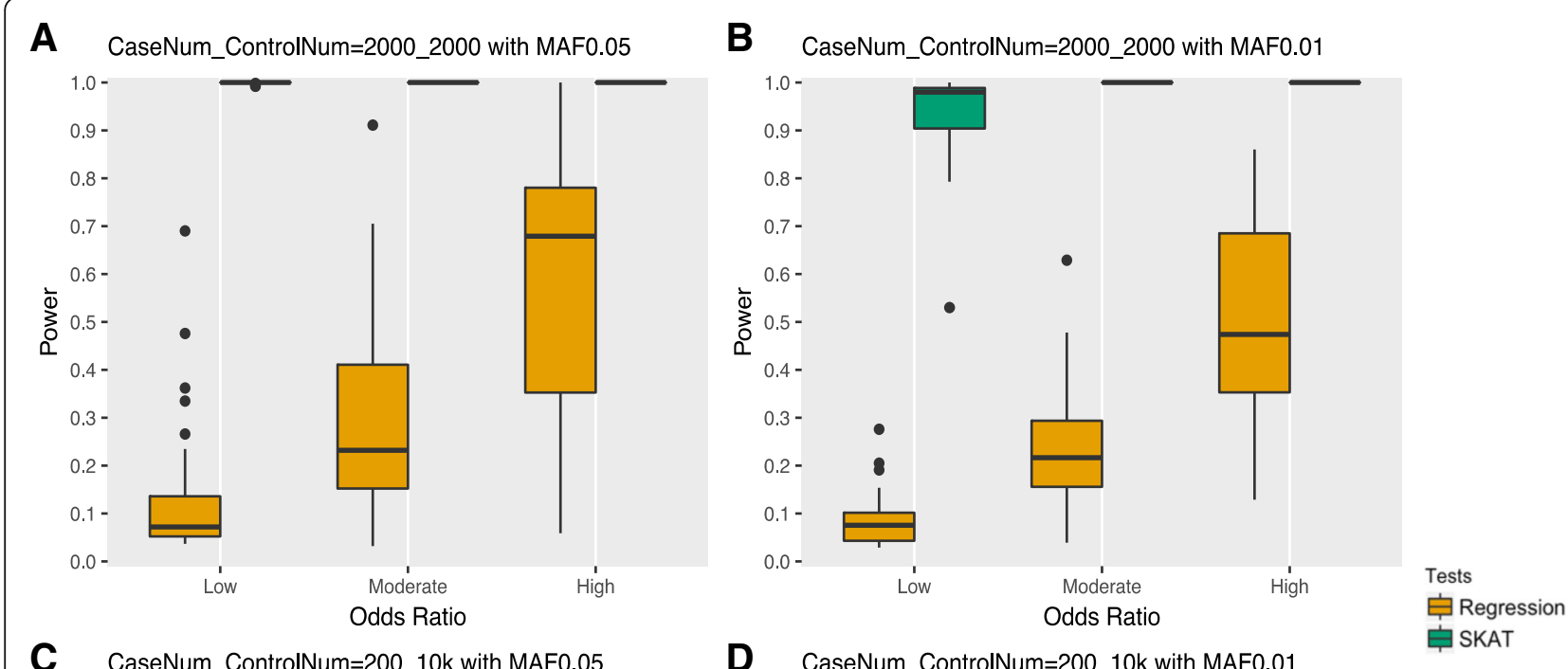

C CaseNum_ControINum=200_10k with MAF0.05

D CaseNum_ControlNum=200_10k with MAF0.01
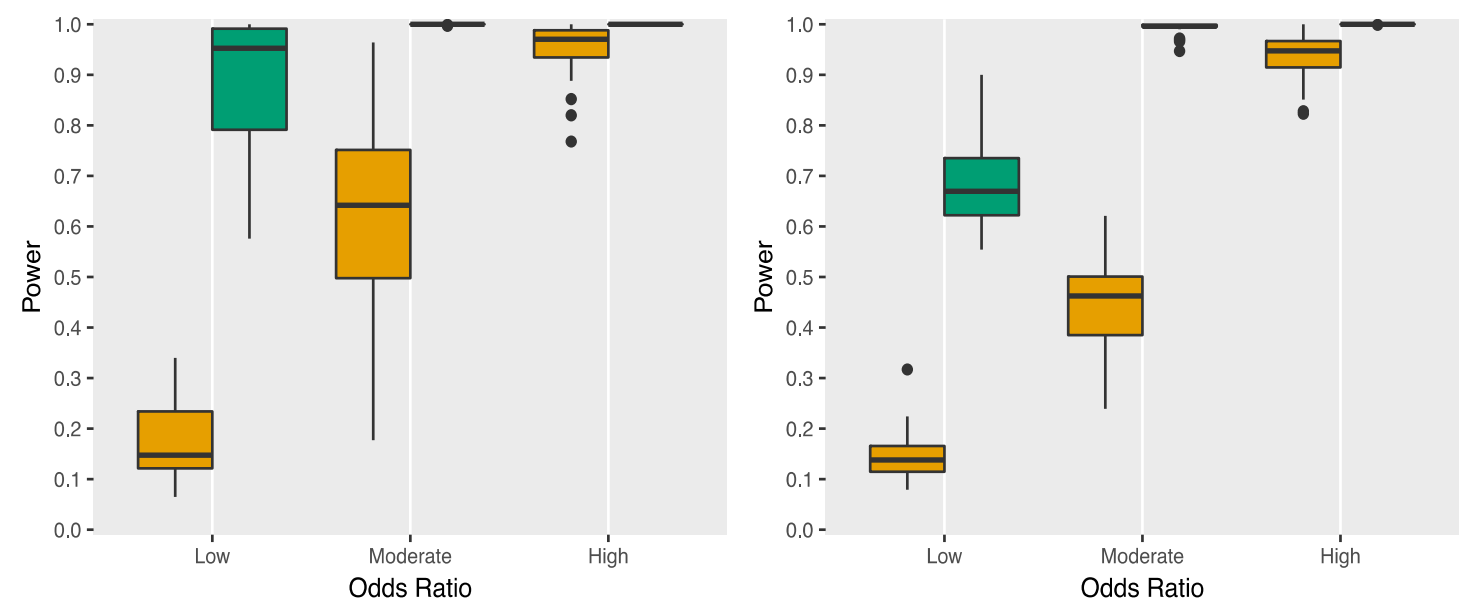

Fig. 3 Power comparison of three models with differing contributions from protective and risk rare genetic variation. The results are shown for variants contributing low, moderate, or high impact on outcome risk or protection. Methods describe the range of odds ratios corresponding to the different categories. (a) Total sample size of 4000 for balanced cases and controls with MAF UB 0.05. (b) Total sample size of 4000 for balanced cases and controls with MAF UB 0.01. (c) 200 cases and 10,000 controls with MAF UB 0.05. (d) 200 cases and 10,000 controls with MAF UB 0.01

2000 controls. However, the opposite trend was observed for SKAT.

\section{Discussion}

Previous simulation studies have been conducted to characterize the statistical performance for burden and dispersion-based approaches using a balanced population of cases and controls [7, 9, 19, 20]. However, there are many scenarios where there may not be balanced case control data for a study, and it is important to know if this will be impactful as rare variant association methods evaluate the joint effect of multiple rare variants between case and control groups. In this study, we sought to evaluate the influence of case control balance on the statistical performance of logistic regression and SKAT rare variant methods.
We found an overall higher type I error rate for unbalanced samples (mostly above 0.05) compared with balanced samples (mostly below 0.05) for both tests, suggesting that an unequal number of cases and controls has a clear statistical impact on type I error for rare variant association analysis. Previous research has reported that the type I error rate for SKAT is conservative for smaller sample sizes [9]. Indeed, our balanced sample size simulations suggest the same trend. However, SKAT has an inflated type I error for unbalanced samples with cases less than 200, thus we recommend researchers interpret those results with caution. Interestingly, regression shows a well-controlled type I error rate for both balanced and unbalanced samples. If controlling type I error is the priority, logistic regression is a more appropriate method than SKAT for both balanced and unbalanced scenarios. 
Statistical power largely depends on the number of disease loci and the odds ratio. In this paper, we evaluated both same-direction signal (i.e. 2.5 odds ratio) and mixed odds ratio models (Table 3 ) on 10 disease loci out of an average of 143 rare variant loci. We assessed the power distribution across various sample sizes using an odds ratio of 2.5. For balanced samples, given that both SKAT and regression have an overall controlled type I error, a total sample size less than 2000 obtains power less than 50\% and more than 4000 obtains power higher than $50 \%$. For unbalanced sample scenarios, SKAT has an overall higher power distribution than regression. Results show that at least 200 case samples are needed to obtain a power of $90 \%$ via SKAT, and an even larger number of cases are required for the regression approach.

As for models with a range of variants contributing to risk and protection for an outcome, our results suggest that SKAT has an overall higher power compared with logistic regression. The results are expected since burden tests lose power when variants contribute to a range of risk and protection for an outcome. Understandably, as the impact of the rare variants on outcome increases, power increases for all scenarios.

Based on our type I error and power results across various unbalanced sample sizes, a clear trend exists between these statistics and the number of cases in addition to the case to control ratio (simulation results of constant case to control ratio are shown in Additional file 1: Figure S3). As many studies ensure the proper case to control ratio, we also recommend that researchers pay attention to the number of cases in the rare variation association studies to help achieve expected type I error and power rates. To our knowledge, our work is the first to propose the landscape of statistics while varying the balance of sample sizes for rare variant association methods.

The likely reason that our simulations present relatively lower power for regression could be a small proportion of disease loci being simulated. As the number of disease loci increases, we expect to observe higher power for burden-based approaches. Future work will aim to simulate various disease loci and odds ratio combinations to provide comprehensive implications on power assessment.

\section{Conclusion}

In this paper, we have presented a simulation study through a wide range of balanced and unbalanced sample sizes, to fully assess the type I error and power distribution for burden and dispersion based rare variant association methods. We observe an impact of sample size imbalance on the statistical performance which can serve as a benchmark for future rare variant analysis.

\section{Methods \\ BioBin}

BioBin is a $\mathrm{C}++$ command line tool that performs rare variant binning and association testing via a biological knowledge driven multi-level approach [29]. The framework of a BioBin analysis is to group rare variants into "bins" based on user-defined biological features followed by statistical tests upon each bin. Biological features, which include genes, inter-genic regions, pathways, and others, are defined by prior knowledge obtained from the Library of Knowledge Integration (LOKI) database [26]. LOKI is a local repository which unifies resources from over thirteen public databases, such as the National Center for Biotechnology dbSNP and gene Entrez database information [31], Kyoto Encyclopedia of Genes and Genomes [32], Pharmacogenomics Knowledge Base [33], Gene Ontology [34], and others. Several select burden and dispersion-based statistical tests have been implemented into BioBin [27, 29], namely linear regression, logistic regression, Wilcoxon rank-sum test, and SKAT [9], which allows users the option of choosing the appropriate test(s). All of the statistical tests have been retained as their original statistical testing framework within BioBin. BioBin also enables users to perform association analysis across multiple phenotypes in a rare variant PheWAS. In this paper, we evaluate power and type I error using both logistic regression and SKAT using the BioBin 2.3.0 software [29]. BioBin software and the user manual are freely available at Ritchie Lab website [35] .

\section{Simulation design}

\section{Sample size and case control ratios}

Simulations were designed to systematically evaluate the impact of different sample sizes, as well as different case control ratios for rare variant association tests. Twelve different scenarios for a balanced number of cases and controls with a total sample size ranging from 20 to 20,000 were simulated. For unbalanced scenarios, a wide range of tests were constructed with case numbers varying from 10 to 7000 and two sets of large control samples $(10,000$ and 30,000$)$. Case to control ratio was calculated as the number of cases divided by the number of controls. Details of the study design with respect to sample size are shown in Table 1 . Moreover, we also designed a few simulations with larger control group (50,000; 100,000; and 200,000), results of which are shown in the Additional file 1: Table S1. Finally, it is important to note that the results would be comparable even if the scenario is reversed and the data include more cases than controls. As long as the customized Madsen and Browning weighting scheme is used, then the results would be the same whether the data include 
Table 1 Simulation Design

Balanced Cases and Controls

Total Sample Size 20, 50, 100, 200, 400, 1000, 2000, 4000, 6000, 10,000, $14,000,20,000$

Unbalanced Cases and Controls

Number of controls 10,000

Number of controls 30,000

Number of cases

$10,25,50,75,85,100,200,500,1000$, $3000,5000,7000$

Number of cases

$10,25,50,75,85,100,200$ 500,1000

1000 cases and 100 controls or 100 cases and 1000 controls (Additional file 1: Figure S4).

\section{Minor allele frequency}

Minor allele frequencies (MAFs) were randomly assigned to our simulated rare variants using allele frequency distribution data from actual whole exome sequencing data from 50,726 patients from the MyCode Community Health Initiative as a part of the DiscovEHR project [36]. Due to the rounding precision of MAF that SeqSIMLA2 [37] requires, we used 0.0015 as the MAF lower boundary to avoid zero MAF for simulated variants. For the MAF upper bound (MAF UB), we simulated two sets of data, one with MAF UB 0.01 and the other with MAF UB 0.05, respectively.

\section{Parameter settings}

As our primary goal is to compare the effect of case-control sample sizes, we set other parameters as constant across all the datasets (Table 2). All simulations were generated with an average of 143 loci per dataset as we calculated this to be the mean number of rare loci from 800 genes in a recent PheWAS study [38]. Here, "locus" refers to a genetic location which harbors genetic variants. We also applied a customized Madsen and Browning [12] weighting scheme as implemented in BioBin for all datasets in order to increase statistical power [27].

\section{Simulation model}

All of the datasets were generated using the software SeqSIMLA2.8, which can be used to design simulated

Table 2 Other Parameter Settings

\begin{tabular}{ll}
\hline Number of Simulations & $\begin{array}{l}1000 \text { runs times } 30 \text { replicates for each } \\
\text { sample size scenario } \\
0.01 \text { and } 0.05\end{array}$ \\
Upper Threshold for MAF & Madsen and Browning [12] \\
Variant Weighting & $5 \%$ \\
Disease Prevalence & 10 \\
Number of Disease Loci & $\begin{array}{l}\text { All disease loci with OR 2.5; Half } \\
\text { of disease loci with risk effect, } \\
\text { the other half with protective effect }\end{array}$ \\
Odds Ratio (OR) &
\end{tabular}

datasets given user-specified sample size, effect sizes for genetic traits, and genetic model [37]. The disease penetrance model in SeqSIMLA is based on a logistic function [37]:

$$
\operatorname{logit}(\mathrm{P}(\text { case }))=\alpha+\beta_{1} \mathrm{x}_{1}+\beta_{2} \mathrm{x}_{2}+\beta_{3} \mathrm{x}_{3}+\ldots+\beta_{\mathrm{p}} \mathrm{x}_{\mathrm{p}}
$$

$\mathrm{x}_{1}, \mathrm{x}_{2}, \mathrm{x}_{3}, \ldots, \mathrm{x}_{\mathrm{p}}$ represent the genotypes across $\mathrm{p}$ disease loci. $\beta_{1}, \beta_{2}, \beta_{3}, \ldots, \beta_{p}$ represent the log of the odds ratios. SeqSIMLA will search for $\alpha$ so that the disease prevalence is close to the specified prevalence. Here, disease prevalence was set to $5 \%$.

\section{Type I error (T1E) and power simulation}

Each type I error or power value was calculated from 1000 independent simulated datasets with significance assessed at $\alpha=0.05$. We replicated 1000 runs 30 times as to account for sampling variability. Running 30 replicates of 1000 datasets was optimal to reduce computational and memory burden. The simulated data did not have any missingness in either genotype or phenotype. Type I error was obtained from null datasets with no genetic association signal. For power, 10 random disease loci with an odds ratio of 2.5 per locus were simulated. In our study, power is defined as the probability of detecting a true signal (i.e. to reject the null hypothesis) when the null hypothesis is false. Power is calculated as the number of datasets that have rejected the null hypothesis at $\alpha=0.05$ level divided by the total number of datasets (i.e. 1000). We also designed three sets of mixed odds ratio models where half of the 10 disease loci had protective effects, and half had risk effects, as described more in the next section.

\section{Mixed odds ratio models}

For most of the simulations, an odds ratio of 2.5 was used for 10 disease loci, indicating consistent risk for all associated rare variants. We also designed three types of protective and risk odds ratio combinations for the 10 disease loci. The detailed odds ratio for 10 disease loci are shown in Table 3, where variants were assigned a range of "Low", "Moderate", or "High" risk or protective impact, randomly. For each mixed model, we calculated protective $(\mathrm{OR}<1)$ effect as the same as the risk effect as to retain the consistent range of association signals.

Table 3 Detailed Parameters for Mixture Odds Ratio Design

\begin{tabular}{llllllllllll}
\hline & \multicolumn{1}{ll}{ Randomly Selected 10} & Disease loci \\
\cline { 2 - 10 } Signal Level & OR $>1$ range (Risk) & \multicolumn{6}{c}{ OR $<1$ range (Protective) } \\
\hline Low & $\mathbf{2 . 3}$ & 2.73 & 3.15 & 3.58 & $\mathbf{4}$ & 0.43 & 0.37 & 0.32 & 0.28 & 0.25 \\
Moderate & $\mathbf{4}$ & 5.25 & 6.5 & 7.75 & $\mathbf{9}$ & 0.25 & 0.19 & 0.15 & 0.13 & 0.11 \\
High & $\mathbf{9}$ & 11.5 & 14 & 16.4 & $\mathbf{1 9}$ & 0.11 & 0.087 & 0.07 & 0.06 & 0.053 \\
\hline
\end{tabular}

Note: The numbers in bold represent the boundaries when selecting the odds ratios 


\section{Boxplot}

All of the boxplots were generated using the "geom_boxplot" function within "ggplot2" R package [39]. The "reshape2" $\mathrm{R}$ package [40] was used for format changing purposes. Each boxplot bar represents the distribution of type I error or power calculated from 30 replicates.

\section{Additional files}

Additional file 1: Figure $\mathbf{S} \mathbf{1}$ and $\mathbf{S 2}$. Type I error and power simulation results with MAF upper bound of 0.05. Figure S3. Type I error and power simulation results using a constant case to control ratio. Figure S4. Type I error comparison when case control sample size is reversed. Table S1. Simulation results for case sample size of 200 and control sample size of $50 \mathrm{k}, 100 \mathrm{k}$ and $200 \mathrm{k}$. (PDF $551 \mathrm{~kb}$ )

Additional file 2: A summary of results for type I error and power simulations with MAF upper bound of 0.01. (XLS $109 \mathrm{~kb}$ )

\section{Abbreviations}

GWAS: Genome-Wide Association Studies; MAF UB: Minor allele frequency upper bound; MAFs: Minor allele frequencies; OR: Odds Ratio; PheWAS: Phenome-Wide Association Studies; SKAT: Sequence Kernel Association Test

\section{Acknowledgements}

We would like to thank Geisinger for providing minor allele frequency information that was obtained from 50,726 patients from the MyCode Community Health Initiative. We would like to thank Dr. Molly Hall, Dr. Anurag Verma and Dr. Shefali Verma for helpful discussions on this project. We would also like to thank Dr. Yogasudha Veturi for the feedback on the manuscript. This work has been presented by Xinyuan Zhang as a poster at American Society of Human Genetics 67th Annual Meeting.

\section{Funding}

This project is funded in part by NIH Al1 16794, Al077505, and under a grant with the Pennsylvania Department of Health (\#SAP 4100070267). The Department specifically disclaims responsibility for any analyses, interpretations or conclusions.

\section{Availability of data and materials}

A summary of results that we have used to generate the figures in the main manuscript is provided in the Additional file 2

\section{Authors' contributions}

$X Z, A O B, S A P$ and MDR conceptualized the project. XZ and MDR led the project. $X Z$ contributed to designing the analysis, performing the analysis and manuscript writing. AOB and SAP assisted with analysis design and provided important feedback on the manuscript. All the authors read and approved the final manuscript.

\section{Ethics approval and consent to participate} Not Applicable.

\section{Consent for publication}

Not Applicable.

\section{Competing interests}

All authors have no conflict of interest to declare.

\section{Publisher's Note}

Springer Nature remains neutral with regard to jurisdictional claims in published maps and institutional affiliations.

\section{Author details}

'Genomics and Computational Biology Graduate Group, Perelman School of Medicine, University of Pennsylvania, Philadelphia, PA, USA. ${ }^{2}$ Department of Biomedical Informatics, Columbia University, New York, NY, USA. ${ }^{3}$ Biomedical and Translational Informatics Institute, Geisinger, Danville, PA, USA. ${ }^{4}$ Department of Genetics, University of Pennsylvania, Perelman School of Medicine, Philadelphia, PA, USA.

Received: 29 August 2018 Accepted: 26 December 2018

Published online: 22 January 2019

\section{References}

1. Pritchard JK. Are rare variants responsible for susceptibility to complex diseases? Am J Hum Genet. 2001;69:124-37.

2. Reich DE, Lander ES. On the allelic spectrum of human disease. Trends Genet. 2001;17:502-10.

3. Cirulli ET, Goldstein DB. Uncovering the roles of rare variants in common disease through whole-genome sequencing. Nature Reviews Genetics. Nature Publishing Group. 2010;11:415-25.

4. Gibson G. Rare and common variants: twenty arguments. Nature Reviews Genetics. Nature Publishing Group. 2012;13:135-45.

5. Zuk O, Schaffner SF, Samocha K, Do R, Hechter E, Kathiresan S, et al Searching for missing heritability: designing rare variant association studies. Proc Natl Acad Sci U S A. 2014;111:E455-64.

6. Manolio TA, Collins FS, Cox NJ, Goldstein DB, Hindorff LA, Hunter DJ, et al. Finding the missing heritability of complex diseases. Nature Nature Publishing Group. 2009;461:747-53.

7. Li B, Leal SM. Methods for detecting associations with rare variants for common diseases: application to analysis of sequence data. Am J Hum Genet. 2008:83:311-21.

8. Lee $S$, Abecasis GR, Boehnke M, Lin X. Rare-variant association analysis: study designs and statistical tests. Am J Hum Genet. 2014;95:5-23.

9. Wu MC, Lee S, Cai T, Li Y, Boehnke M, Lin X. Rare-variant association testing for sequencing data with the sequence kernel association test. Am J Hum Genet. 2011:89:82-93.

10. Morgenthaler S, Thilly WG. A strategy to discover genes that carry multiallelic or mono-allelic risk for common diseases: a cohort allelic sums test (CAST). Mutat Res. 2007;615:28-56.

11. Han F, Pan W. A data-adaptive sum test for disease association with multiple common or rare variants. Hum Hered. 2010;70:42-54.

12. Madsen BE, Browning SR. A groupwise association test for rare mutations using a weighted sum statistic. PLoS Genet. 2009;5:e1000384.

13. Hoffmann TJ, Marini NJ, Witte JS. Comprehensive approach to analyzing rare genetic variants. PLoS ONE. Public Libr Sci. 2010;5:e13584.

14. Neale BM, Rivas MA, Voight BF, Altshuler D, Devlin B, Orho-Melander M, et al. Testing for an unusual distribution of rare variants. PLoS Genet. 2011;7: e1001322.

15. Lee $\mathrm{S}, \mathrm{Wu} M C$, Lin $\mathrm{X}$. Optimal tests for rare variant effects in sequencing association studies. Biostatistics. 2012;13:762-75.

16. Derkach A, Lawless JF, Sun L. Robust and powerful tests for rare variants using Fisher's method to combine evidence of association from two or more complementary tests. Genet. Epidemiol. Wiley-Blackwell. 2013:37:110-21.

17. Sun J, Zheng Y, Hsu L. A unified mixed-effects model for rare-variant association in sequencing studies. Genet Epidemiol Wiley-Blackwell. 2013;37: 334-44.

18. Chen LS, Hsu L, Gamazon ER, Cox NJ, Nicolae DL. An exponential combination procedure for set-based association tests in sequencing studies. Am J Hum Genet. 2012;91:977-86.

19. Basu S, Pan W. Comparison of statistical tests for disease association with rare variants. Genet. Epidemiol. Wiley-Blackwell. 2011;35:606-19.

20. Bacanu S-A, Nelson MR, Whittaker JC. Comparison of statistical tests for association between rare variants and binary traits. PLoS ONE. Public Libr Sci. 2012;7:e42530

21. Bush WS, Oetjens MT, Crawford DC. Unravelling the human genomephenome relationship using phenome-wide association studies. Nature Reviews Genetics Nature Publishing Group. 2016;17:129-45.

22. Verma A, Ritchie MD. Current scope and challenges in phenome-wide association studies. Curr Epidemiol Rep Springer International Publishing. 2017:4:321-9.

23. Denny JC, Ritchie MD, Basford MA, Pulley JM, Bastarache L, Brown-Gentry K, et al. PheWAS: demonstrating the feasibility of a phenome-wide scan to discover gene-disease associations. Bioinformatics. 2010;26:1205-10.

24. Pendergrass SA, Ritchie MD. Phenome-wide association studies: leveraging comprehensive phenotypic and genotypic data for discovery. Curr Genet Med Rep Springer US. 2015;3:92-100. 
25. Bansal V, Libiger O, Torkamani A, Schork NJ. Statistical analysis strategies for association studies involving rare variants. Nature Reviews Genetics. Nature Publishing Group. 2010;11:773-85.

26. Moore CB, Wallace JR, Frase AT, Pendergrass SA, Ritchie MD. BioBin: a bioinformatics tool for automating the binning of rare variants using publicly available biological knowledge. BMC Med Genomics. 2013;6 Suppl 2:S6.

27. Basile $A O$, Wallace $J R$, Peissig P, McCarty CA, Brilliant M, Ritchie MD. Knowledge driven binning and PheWAS analysis in Marshfield personalized medicine research project using BioBin. Pac Symp Biocomput NIH Public Access. 2016;21:249-60.

28. Moore CB, Wallace JR, Frase AT, Pendergrass SA, Ritchie MD. Using BioBin to explore rare variant population stratification. Pac Symp Biocomput. NIH Public Access. 2013:332-43.

29. Basile AO, Byrska-Bishop M, Wallace J, Frase AT, Ritchie MD. Novel features and enhancements in BioBin, a tool for the biologically inspired binning and association analysis of rare variants. Bioinformatics. 2018;34:527-9.

30. Moore CB, Basile AO, Wallace JR, Frase AT, Ritchie MD. A biologically informed method for detecting rare variant associations. BioData Min. BioMed Central. 2016;9:27.

31. Wheeler DL, Church DM, Federhen S, Lash AE, Madden TL, Pontius JU, et al. Database resources of the National Center for biotechnology. Nucleic Acids Res. Oxford University Press. 2003;31:28-33.

32. Kanehisa M. KEGG: Kyoto encyclopedia of genes and genomes. Nucleic Acids Res. 2000;28:27-30.

33. Hewett M, Oliver DE, Rubin DL, Easton KL, Stuart JM, Altman RB, et al. PharmGKB: the pharmacogenetics Knowledge Base. Nucleic Acids Res Oxford University Press. 2002;30:163-5.

34. Gene Ontology Consortium. The gene ontology (GO) database and informatics resource. Nucleic Acids Res. 2004;32:258D-261.

35. BioBin software. https://ritchielab.org/software/biobin-download. Accessed 18 Dec 2018.

36. Dewey FE, Murray MF, Overton JD, Habegger L, Leader JB, Fetterolf SN, et al. Distribution and clinical impact of functional variants in 50,726 wholeexome sequences from the DiscovEHR study. Science. 2016;354 aaf6814.

37. Chung R-H, Tsai W-Y, Hsieh C-H, Hung K-Y, Hsiung CA, Hauser ER. SeqSIMLA2: simulating correlated quantitative traits accounting for shared environmental effects in user-specified pedigree structure. Genet. Epidemiol. Wiley-Blackwell. 2015;39:20-4.

38. Verma SS, Josyula N, Verma A, Zhang X, Veturi Y, Dewey FE, et al. Rare variants in drug target genes contributing to complex diseases, phenomewide. Sci Rep. Nat Publ Group. 2018;8:4624.

39. Gómez-Rubio V. ggplot2- Elegant Graphics for Data Analysis (2nd Edition). J. Stat. Softw. 2017:77:1-3.

40. Wickham H. Reshaping data with the reshapePackage. J Stat Softw. 2007;21: $1-20$

Ready to submit your research? Choose BMC and benefit from:

- fast, convenient online submission

- thorough peer review by experienced researchers in your field

- rapid publication on acceptance

- support for research data, including large and complex data types

- gold Open Access which fosters wider collaboration and increased citations

- maximum visibility for your research: over $100 \mathrm{M}$ website views per year

At $\mathrm{BMC}$, research is always in progress.

Learn more biomedcentral.com/submissions 